\title{
GRAPPA Trainees Symposium 2013: A Report from the GRAPPA 2013 Annual Meeting
}

\author{
Agnes Szentpetery, Mary Ann N. Johnson, and Christopher T. Ritchlin
}

\begin{abstract}
At the 2013 annual meeting of the Group for Research and Assessment of Psoriasis and Psoriatic Arthritis (GRAPPA) in Toronto, Ontario, Canada, 26 dermatology and rheumatology fellows engaged in psoriasis or psoriatic arthritis research were invited to present their work at the Trainees Symposium, which preceded the annual meeting and was also attended by GRAPPA members from around the world. Herein, we provide a brief overview of the 6 oral presentations and 25 posters presented, which reflect the focus and diversity of current basic and clinical research in psoriatic disease. (J Rheumatol 2014;41:1200-5; doi:10.3899/jrheum.140170)
\end{abstract}

\author{
Key Indexing Terms: \\ PSORIATIC ARTHRITIS \\ DERMATOLOGIST
}

PSORIASIS

TRAINEE

\section{RHEUMATOLOGIST \\ GRAPPA}

The sixth Trainees Symposium was held at the 2013 annual meeting of the Group for Research and Assessment of Psoriasis and Psoriatic Arthritis (GRAPPA) in Toronto, Ontario, Canada. A total of 26 rheumatology or dermatology trainees from over 10 countries in North America, South America, and Europe participated in the symposium, which has become one of the highlights of the annual GRAPPA meetings ${ }^{1,2,3,4}$. Trainees who are current members of GRAPPA or who were nominated by GRAPPA members were invited to submit abstracts based on recent research in psoriatic arthritis (PsA) or psoriasis (PsO). At the 2013 meeting, 40 abstracts were submitted and ranked by a committee of reviewers. Five trainees with the highest-scored abstracts were invited to deliver oral presentations (1 trainee presented twice); the remainder presented posters that outlined key aspects of their research.

The session was chaired by Christopher Ritchlin (Rochester, New York, USA) with an audience of about 100 GRAPPA members, who queried the trainees and provided feedback on how to transition current research projects to the next level.

\footnotetext{
From the Department of Rheumatology, St. Vincent's University Hospital, Dublin, Ireland; University of California Davis, Department of Dermatology, Davis, California; and Division of Allergy, Immunology, and Rheumatology, University of Rochester Medical Center, Rochester, New York, USA.
}

A. Szentpetery, MD, Department of Rheumatology, St. Vincent's University Hospital; M.A.N. Johnson, MD, University of California Davis, Department of Dermatology; C.T. Ritchlin, MD, MPH, Professor of Medicine, Division of Allergy, Immunology, and Rheumatology, University of Rochester Medical Center.

Drs. Szentpetery and Johnson contributed equally to this manuscript. Address correspondence to Dr. Ritchlin, Professor of Medicine, Allergy, Immunology, and Rheumatology Division, Center for Musculoskeletal Research, University of Rochester Medical Center, 601 Elmwood Ave., Box 695, Rochester, New York 14642, USA.

E-mail: christopher_ritchlin@urmc.rochester.edu

\section{Oral Presentations}

Feasibility, reliability, and sensitivity to change of 4 radiographic scoring methods in patients with PSA (William Tillett, Bath, UK)

Dr. Tillett presented results from a study of 4 radiographic scoring methods that are currently used to evaluate radiographic damage as an outcome measure in PsA ${ }^{5}$. Hand and feet radiographs from 50 patients with PsA were scored at 2 timepoints, with each of the modified Steinbrocker score (STB), Modified Sharp Score (MSS), Sharp/van der Heijde Modified Method (VDH), and PsA Ratingen Score (PARS) methods by 2 assessors. Radiographs of 10 patients were scored for reliability using intraclass correlation coefficients (ICC). Sensitivity to change was estimated using a standardized response mean (SRM) and smallest detectable change (SDC).

Patients' mean age was 50 years, disease duration 10 years, and followup 25 months. Intrarater reliability was excellent for all methods (ICC > 0.97). Interrater reliability was highest for the VDH (ICC 0.95-0.99). The SDC for the STB, PARS, MSS, and VDH methods was $2.9 \%, 2.1 \%$, $1.4 \%$, and $1.2 \%$, respectively; and the SRM were $0.46,0.44$, 0.77 , and 0.79 , respectively. The mean time to score each of the scoring methods was $6.2,10.5,14.6$, and $14.4 \mathrm{~min}$, respectively.

Dr. Tillett concluded that the VDH instrument is the most reliable and sensitive to change, but it took longer to perform. The STB is the most feasible but lacks the sensitivity of the VDH. The SDC of the PARS is close to that of the VDH but is quicker to perform, and inclusion of proliferation may be more specific to PsA.

Periarticular bone gain in early PsA but not in rheumatoid arthritis (RA) following antirheumatic treatment assessed by digital x-ray radiogrammetry (Agnes Szentpetery, Dublin, Ireland) 
Dr. Szentpetery presented results from a prospective study evaluating changes in periarticular bone mineral density as measured by digital $x$-ray radiogrammetry (DXR-BMD) in early PsA and patients with RA prior to, and at 3 and 12 months after introducing an antirheumatic drug. Mean DXR-BMD values of both hands and changes in DXR-BMD ( $\left.\mathrm{mg} / \mathrm{cm}^{2} / \mathrm{month}\right)$ were compared between the 2 groups at all timepoints.

Sixty-four (32 PsA, $32 \mathrm{RA})$ recent-onset (<12 mos), active, treatment-naive patients were selected with median age 43 years. Ninety-five percent of the patients began taking a disease-modifying antirheumatic drug (DMARD), and $12 \%$ were also started on a tumor necrosis factor inhibitor (anti-TNF). DXR-BMD decreased in both diseases at 3 months and was lower in RA at 12 months $(\mathrm{p}=0.004)$ compared to baseline. In contrast, DXR-BMD increased in PsA over 12 months. DXR-BMD loss was significantly higher in RA compared to PsA from baseline to 12 months.

Study results were unique in showing hand bone loss as early as 3 months, as measured by DXR in both PsA and RA despite therapeutic intervention with an antirheumatic drug. After 1 year of treatment, cortical bone gain was observed in PsA but bone loss progressed in RA, supporting the hypothesis of different pathomechanisms underlying hand bone remodeling in PsA and RA.

GC-TOF MS analysis reveals metabolite difference in PSO and PsA patients compared to healthy individuals (Mary Ann N. Johnson, Davis, California, USA)

Dr. Johnson presented results from a study exploring the metabolite profiles of patients with $\mathrm{PsO}$ without $\mathrm{PsA}$, PsO with PsA, and healthy controls. Gas chromatography time-of-flight mass spectrometry (GC-TOF MS) was used to compare the metabolite profiles of 30 human sera samples collected from 3 groups: patients with skin-limited $\mathrm{PsO}$, patients with both PsO and PsA, and healthy controls. Metabolic measurements were analyzed using univariate statistics, multivariate modeling with adjustment for multiple comparisons, and biochemical/chemical similarity network analysis.

When comparing the metabolite profiles of the 3 groups, significant alterations in glucuronic acid, arabinose, phosphoric acid, and lignoceric acid were observed. When comparing the metabolite profiles of patients with PsO and control patients, significant differences were characterized by a decrease in $\alpha$-ketoglutaric acid and an increase in asparagine, glutamine, and $\beta$-sitosterol in patients with $\mathrm{PsO}$ compared to controls.

These findings may indicate differences in cellular metabolism and cholesterol absorption in PsO, PsA, and healthy individuals.

Characteristics and predictors of minimal disease activity
$(M D A)^{6}$ in patients with PsA taking anti-TNF (Amir Haddad, Toronto, Ontario, Canada)

In a study conducted by Dr. Haddad, 305 patients treated with anti-TNF were recruited and followed at 3-6 month intervals from a single center cohort. Of 226 patients who were in non-MDA state, 146 (63\%) achieved MDA after an average of $1.3 \pm 1.5$ years and for a mean duration of $3.46 \pm$ 2.2 years. Among patients who achieved MDA, at baseline more were male, younger at the age of diagnosis, and more likely to have clinical damage and axial involvement compared to the non-MDA group. Patients with non-MDA had higher body mass index (BMI) at baseline, were more frequently classified functional class III/IV, and had lower Medical Outcome Study Short Form-36 (SF-36) scores. The majority of patients in both groups were also treated with DMARD and nonsteroidal antiinflammatory drugs. The survival analysis showed that after adjusting at each visit for sex, age, duration of disease, abnormal erythrocyte sedimentation rate (ESR), Psoriasis Area and Severity Index (PASI), obesity, and active joint count (AJC), only AJC (OR $=0.70$, CI $0.57-0.88, \mathrm{p}=0.002)$ and PASI score $(\mathrm{OR}=0.84, \mathrm{CI}$ $0.72-0.99, \mathrm{p}=0.03$ ) lowered the odds of achieving MDA.

In this analysis, only lower AJC and PASI score were predictors of response to treatment with TNF blockers.

Anticitrullinated peptide antibody (ACPA) in patients with PsA: clinical relevance (DoQuyen Huynh, San Diego, California, USA)

In 2012, Dr. Huynh compared the clinical features between ACPA-positive and ACPA-negative patients with PsA in a cross-sectional study of 5363 patients with physician-diagnosed PsA in the CORRONA (Consortium of Rheumatology Researchers of North America) database.

Of $17.7 \%$ patients with test results for ACPA, 842 were ACPA- and 116 ACPA+. Patients were evaluated for tender joint count (TJC), swollen joint count (SJC), health assessment questionnaire (HAQ), patient global assessment, physician global assessment, 28-joint Disease Activity Score (DAS28), Composite Disease Activity Index (CDAI), presence of enthesitis or dactylitis, skin psoriasis activity, presence of Sjögren symptoms, and radiographic changes. Both groups were assessed for the presence of rheumatoid factor (RF), and its contribution in any differences. No significant differences were observed in any measures of disease activity or the use of immunomodulatory medications between ACPA-positive and ACPA-negative patients. ACPA-positive patients had a lower average TJC (mean difference -0.89 ; $95 \%$ CI $-1.78,-0.02$ ). Further stratification into subgroups based upon the presence of RF did not reveal any clinically important differences.

Dr. Huynh suggested that in contrast to RA, the presence of positive ACPA may represent an epiphenomenon in PsA.

Patient involvement in the development of PsA domain Personal non-commercial use only. The Journal of Rheumatology Copyright @ 2014 . All rights reserved. 
construct and outcome measures; a systematic "pearl-growing” review (William Tillett, Bath, UK)

In a literature review, Dr. Tillett performed a search of Medline, Embase, and Cochrane database (1970 to January 3,2013 ) for the following keywords: "psoriatic arthritis" in combination with (AND) "domain" OR "outcome" OR "assessment" OR "validation" (AND) "composite measure," "physical function," "skin activity," "patient global," "pain," "health-related quality of life," "peripheral joint activity," "enthesitis," "dactylitis," "fatigue," "nails," "physician global," "spinal," "participation."

Of 1238 articles reviewed, 63 were assessed using a literature evaluation tool based upon the European League Against Rheumatism guidelines for patient involvement in research. Six articles described patient involvement. Only 1 outcome measure, the PsA Quality of Life (PsAQoL), described patient involvement during the initial development stage and 2 studies involved patients in the assessment of existing measures. Three articles reported the involvement of 4 patients during the development of the Outcome Measures in Rheumatology (OMERACT) core set for PsA.

This review confirms that much of the original domain construct, outcome measures, and disease activity indices were developed without substantial patient input. The authors propose to convene a GRAPPA Patient Involvement in Outcome Measures group to determine the next steps for increased patient involvement in reviewing the domain construct of PsA and the further assessment of outcome measures.

\section{Poster Presentations}

Muhammad Haroon (Dublin, Ireland) performed clinical phenotyping of PsA cases to characterize the clinical features associated with particular HLA class I alleles and their haplotypes. A consecutive cohort of 283 patients with PsA, previously enrolled in a genetic study, was included ${ }^{7}$. Four traits (sacroiliitis, enthesitis, joint deformity, and dactylitis) were analyzed. Mean age of patients was $55 \pm 12$ years, PsA duration $19 \pm 9$ years, $60 \%$ required anti-TNF, $25 \%$ had sacroiliitis, $44.5 \%$ radiographic erosions, and $53 \%$ dactylitis.

Higher sacroiliitis risk alleles/haplotypes $(\mathrm{a} / \mathrm{h})$ were $\mathrm{B} * 08: 01, \mathrm{~B} * 27: 05$, and $\mathrm{C}^{*} 05$ not in a $\mathrm{B} * 44-\mathrm{C} * 05$ haplotype. For enthesitis, $\mathrm{B} * 27: 05, \mathrm{C}^{*} 01: 02$, and $\mathrm{C}^{*} 05$ not in a $\mathrm{B} * 44-\mathrm{C}^{*} 05$ haplotype were higher enthesitis risk $\mathrm{a} / \mathrm{h}$. Higher dactylitis risk a/h were $\mathrm{B} * 27: 05, \mathrm{C}^{*} 02, \mathrm{~B} * 08$, and

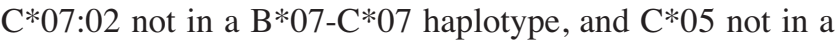
$\mathrm{B} * 44$ haplotype. Higher joint deformity risk a/h were $\mathrm{B} * 08$, C*07:01, and $C^{*} 05$ not in a $\mathrm{B} * 44$ haplotype.

This research provided evidence that HLA genotype importantly influences the clinical phenotype of PsA, and that these phenotypes are likely the result of cis and trans interactions of multiple individual alleles.
Dr. Haroon also presented results of a study that evaluated the prevalence and clinical associations of metabolic syndrome (MetS) in an ethnically homogenous consecutive cohort of 283 patients. MetS was present in $44 \%$ of the cohort; $50 \%$ of these patients with established PsA had clustering of $\geq 4$ MetS risk factors. Mean BMI was $29 \pm 5$. Patients with MetS had more type-2 PsO, later PsO and PsA age of onset, shorter time from $\mathrm{PsO}$ to arthritis development, and higher HAQ, fatigue, and EQ-5D scores. This study emphasized that MetS is highly prevalent in PsA and is associated with the severity of underlying psoriatic disease.

Michele Gilio (Naples, Italy) compared 60 younger-onset (< 60 years) and 16 late-onset ( $\geq 60$ years) early PsA patients with symptom duration less than 52 weeks to evaluate the influence of the age at the disease onset on clinical and laboratory variables. Sensitivity of the ClASsification for Psoriatic ARthritis criteria (CASPAR) and disease characteristics were found to be similar in the 2 cohorts.

Laura Acosta Felquer (Buenos Aires, Argentina) constructed an ultrasound (US) scoring system including 3 structures: joints, tendons, and entheses (16, 8, and 4 sites, respectively) to assess treatment response 3 months after initiating or changing a DMARD or anti-TNF agent. Twenty-six patients with mean disease duration of 3 years were recruited. All clinical variables improved at 3 months [ESR, C-reactive protein (CRP), TJC, SJC, patients' pain, and disease activity visual analog scale (VAS), HAQ, DAS28, CDAI, Simplified DAI, PsA Screening Evaluation, PASI, Composite Psoriatic Disease Activity Index, Bath Ankylosing Spondylitis DAI (BASDAI)] with the exception of Leeds Enthesitis Index. US findings correlated with clinical disease activity, and US score $\geq 10$ showed $75 \%$ sensitivity and $64 \%$ specificity for non-MDA. The authors propose a larger study to validate the scoring system for treatment response in short interval.

Ari Polachek (Tel Aviv, Israel) conducted a study investigating the efficacy and safety of vaccination against influenza virus among patients with $\mathrm{PsA}$ and $\mathrm{PsO}$. Sixty-three patients with PsA, 4 with PsO, and 30 healthy controls were vaccinated using the Sanofi Pasteur vaccine recommended by the World Health Organization in 2012. The response rate to the influenza vaccine was not affected by age, sex, disease activity, or the use of anti-TNF or DMARD. No significant changes were observed in disease activity measures. Temporary local vaccination-site inflammation was the most common adverse effect in both groups. This study demonstrated that vaccination against seasonal influenza is safe and induces an appropriate response in patients with PsA and PsO, similarly to healthy controls.

Penélope Esther Palominos (Paris, France) investigated the effect of PsA on patients' quality of life (QOL). Pain, functional disability, social discrimination in relation to

Personal non-commercial use only. The Journal of Rheumatology Copyright @ 2014 . All rights reserved. 
$\mathrm{PsO}$, fatigue, and anxiety were found to be the most important domains from patients' perspective. The authors suggest incorporating emotional and social aspects of the disease into outcome measures of PsA.

Jenny Paola Varela (Bogotá, Colombia) examined whether including radiographic evidence of periarticular new bone formation (NBF) would change the diagnostic performance of the CASPAR criteria. Of 72 patients with $\mathrm{PsO}, 25$ were diagnosed with PsA (24 had periarticular and 7 juxtaarticular NBF). Although periarticular NBF had better sensitivity for discriminating PsA than juxtaarticular $\mathrm{NBF}$, there was no difference in sensitivity, specificity, and predictive values when included in the CASPAR criteria.

Cindy J. Chambers (Davis, CA, USA) conducted an epidemiologic study investigating sexual behavior in patients with PsO. The authors used the National Health and Nutrition Examination Survey data from 2003 to 2006 (women and men) and 2009 to 2010 (men only) using responses from dermatology and sexual behavior questionnaires. PsO was not associated with differences in sexual orientation among women or men. Heterosexual women with $\mathrm{PsO}$ had 1.13 times more unprotected sex than women without $\mathrm{PsO}$. This study highlighted the importance of involving the patients' perspectives regarding their sexual health and the potential effect of $\mathrm{PsO}$ on sexual behavior.

Abhijeet Danve (Portland, Oregon, USA) retrospectively compared disease characteristics and QOL in patients with PsA with axial involvement (PsAax, $\mathrm{n}=28$ ), PsA with peripheral disease $(n=26)$, and patients with axial spondyloarthritis without PsO (axSpA, $n=29)$. Mean age, disease duration, BMI, BASDAI, and Routine Assessment of Patient Index Data (RAPID3) scores were similar among the 3 groups. PsAax patients had significantly higher prevalence of peripheral arthritis compared to those with axSpA alone. Uveitis was less frequent in PsA compared to axSpA patients. Depression was more prevalent in patients with PsO, suggesting an important effect on QOL related to skin disease.

Rana Aslanov (St. John's, Newfoundland, Canada) presented results of a study evaluating the prevalence of malignancies in patients with early $(n=84)$ and established $(n=112)$ PsA, defined as $<2$ and $\geq 2$ years from diagnosis, respectively. Among the 196 patients, 9.7\% had a malignancy; the most frequently observed cancers were cervical, bowel, and lung. Patients with established disease were significantly younger at the onset of PsO and PsA, and had an increased prevalence rate of malignancies compared to early PsA.

Carrillo Ignacio (Buenos Aires, Argentina) studied whether measures of disease activity, physical function, and the presence of radiographic sacroiliitis correlate with BASDAI in patients with PsA. Seventy patients with median disease duration of 7.3 years were included. BASDAI scores correlated with RAPID3, Bath AS
Functional Index, HAQ, Disease Activity Index for PsA, and DAS28, and were significantly higher in patients with sacroiliitis compared to patients without sacroiliitis.

Lihi Eder (Toronto, Ontario, Canada) examined the usefulness of carotid atherosclerosis assessment in cardiovascular risk stratification of patients with psoriatic disease compared to the Framingham Risk Score (FRS) in 127 patients with $\mathrm{PsA}$ and 104 with $\mathrm{PsO}^{8}$. Carotid intima-media thickness (cIMT) and total plaque area (TPA) were measured by US. Patients were stratified into 3 US-based risk categories according to the severity of atherosclerosis and were classified into 3 FRS-based risk categories (low, intermediate, and high). FRS correlated moderately with TPA and cIMT. Of patients with FRS-based low to intermediate risk, 57.5\% had carotid plaques. Patients with PsA tended to be classified into a higher US-based risk category $(\mathrm{p}=0.04)$ as were patients who used DMARD and anti-TNF. The authors proposed that US assessment of subclinical atherosclerosis may improve risk stratification of patients with psoriatic disease, particularly those with PsA.

Agnes Szentpetery (Dublin, Ireland) investigated clinical markers predictive for radiographic progression over 1 year in 32 recent-onset patients with active PsA, of whom 57.2\% took a DMARD only, and $42.8 \%$ were taking a DMARD and anti-TNF at 12 months. Hand and foot radiographs were scored using the VDH modified method for PsA at baseline and 1 year. Multivariate analysis demonstrated that elevated CRP at baseline is associated with radiographic damage in patients treated with a DMARD only (OR 2.51, $\mathrm{p}=0.025)$. High TJC28 was found to be a strong risk factor for radiographic progression in patients with PsA.

Anna R. Moverley (Leeds, UK) interviewed patients with PsA to develop a definition of flare from the patient's perspective. Six overarching themes pertaining to flare were identified: physical and psychological symptoms, fatigue, loss of normal function, social withdrawal, and patient's interpretation of flare. Study results emphasized that flare in PsA is more than an SJC or TJC as measured in clinical practice. The authors propose to continue this work by observing core domains of flare to establish agreement between patients and healthcare professionals on a definition of flare in PsA.

Ulku Ucar (Antalya, Turkey) measured serum inter-leukin 17A (IL-17A), IL-17B, IL-17C, IL-17F, and IL-23 levels in 40 patients with PsA and in 27 healthy controls by ELISA to explore their relationship with clinical and laboratory findings. Results showed inverse correlations between IL-17C levels and pain and general health VAS scores. IL-17A levels were higher in PsA compared to healthy subjects $(p=0.012)$, while IL-17C levels were elevated in healthy controls $(\mathrm{p}=0.003)$ and among smokers.

Dr. Ucar also presented results from a study where the premise was that impairment of sleep and QOL are reported symptoms in PsA. Forty patients with PsA were evaluated

Personal non-commercial use only. The Journal of Rheumatology Copyright @ 2014 . All rights reserved. 
by PSAQol, Nottingham Health Profile, and Maastricht Ankylosing Spondylitis Enthesitis Score. Only QOL correlated with sleep quality in PsA.

In a third poster, Dr. Ucar assessed the ability of the Duruöz Hand Index (DHI) to evaluate functional disability of hands in patients with PsA. DHI was found to be a reliable and valid instrument to evaluate functional disability of the hand.

Zahi Touma (Toronto, Ontario, Canada) conducted a retrospective analysis to explore the ability of patient-reported outcomes (PRO) such as Dermatology Life Quality Index, Functional Assessment of Chronic Illness Therapy, EQ-5D, Fatigue Severity Scale, HAQ, and SF-36 in assessing responsiveness in PsA after initiation of a biological treatment. Of 223 patients with active disease followed for 1.18 year, 70 were stratified as improved, 126 same, and 27 worsened based on patient global assessment. Responsiveness was noticeable in the patients who improved and patients who worsened. Study results demonstrated that PRO are responsive and valid measures having the ability to identify improvement and worsening in response to treatment.

Colombres Francisco (Buenos Aires, Argentina) studied the prevalence of early PsA in a cohort of patients with early spondyloarthritis from the Consortium of Early Spondyloarthritis project. Of 125 patients included, 37\% were diagnosed with PsA. All patients had $\mathrm{PsO}$ vulgaris with time of onset prior to diagnosis 32 months (1-360). Time from $\mathrm{PsO}$ to arthritis development was $16 \pm 10$ months. The frequency of HLA-B27 positivity in patients with PsA was lower than that reported in other series.

Shawn Rose (Philadelphia, Pennsylvania, USA) conducted a pilot study evaluating vascular inflammation in patients with $\mathrm{PsO}$ and PsA, and the association with sacroiliitis using a whole-body fluorodeoxyglucose (FDG) positron emission tomography/computed tomography (PET/CT). Mean and maximum standard uptake values (SUV) on FDG-PET/CT of the subchondral and periarticular sacroiliac (SI) joints and the aorta were measured to determine the extent of inflammation. Sacroiliitis was higher in PsA than in PsO. After adjustment for cardiovascular disease (CVD) risk factors, SUV values of the SI joints were associated with aortic inflammation $(\mathrm{p}<0.001)$. These data suggest that sacroiliitis may identify patients at increased risk for CVD.

Dr. Johnson investigated atrial fibrillation (AF) and electrocardiographic characteristics among 169 patients with $\mathrm{PsO}$ and coronary artery disease (CAD), matched to patients without PsO. FRS was calculated for AF to predict incident AF. A second cohort of 2078 patients with PsO was matched to 6234 patients without PSO. The investigators reported no statistically significant difference in the electrocardiographic characteristics or the 5-year incidence of AF among patients with $\mathrm{PsO}$ versus matched controls.
Multivariable analysis revealed no association between $\mathrm{PsO}$ of varying severity and incident AF.

Neal Kumar (Boston, Massachusetts, USA) conducted a literature review of studies published on the utility of teledermatology for PsO and PsA by searching original articles in the PubMed database from 1980 to January 2013. The review showed that teledermatology is a cost-effective and reliable tool in the management of $\mathrm{PsO}$.

Gagan D. Singh (Sacramento, California, USA) presented a retrospective analysis of 65 patients with PsA (mean length of diagnosis prior to angiography, 13.8 years) and CAD who underwent coronary angiography from 2006 to 2011 at the University of California Davis Medical Center. Patients with PsA have significantly increased disease extent and stenosis severity of CAD compared to age- and sex-matched controls. These results suggest that chronic inflammation may predispose PsA patients to more diffuse and severe degrees of CAD.

Ana-Maria Orbai (Baltimore, Maryland, USA) presented preliminary results on the Patient Reported Outcome Measurement Information System (PROMIS) use in a PsA clinic. A questionnaire was administered on a touch-screen device to 22 participants at their routine visit. PROMIS measures of pain, physical function, fatigue, sleep disturbance/impairment, anger, anxiety, depression, participation, and satisfaction with social roles appeared to be different in patients reporting flare compared to patients with no flare. The authors will evaluate the validity of these measures in larger numbers of patients.

Jane Park (Seattle, Washington, USA) described efforts of the GRAPPA Definition of Inflammatory Musculoskeletal Disease Project to develop practical clinical criteria to define inflammatory arthritis, enthesitis, dactylitis, and spondylitis for PsA. The expected outcome is simple algorithmic criteria to reliably identify inflammatory musculoskeletal disease to help clinicians more accurately apply CASPAR criteria to these disease symptoms in PsA.

Both the oral and poster presentations by trainees generated lively discussion with the GRAPPA faculty. A ceremony was held during the gala dinner when prizes for the best abstracts were presented by Prof. John Moll (retired, previously Leeds, UK), with the first prize awarded to Dr. Muhammad Haroon from Dublin, Ireland. The next GRAPPA Trainees Symposium will be held in July 2014 in New York City, USA.

\section{REFERENCES}

1. Coates LC, Ritchlin CT. GRAPPA Trainees Symposium 2009: a report from the GRAPPA 2009 annual meeting. J Rheumatol 2011;38:526-9.

2. Ritchlin CT. GRAPPA Trainees Symposium 2010: a report from the GRAPPA 2010 annual meeting. J Rheumatol 2012;39:394-7.

3. Ash Z, Ritchlin CT. GRAPPA Trainees Symposium 2011: a report from the GRAPPA 2011 annual meeting. J Rheumatol 2012;39:2184-8.

4. Garg N, Touma Z, Ritchlin CT. GRAPPA Trainees Symposium 2012: a report from the GRAPPA 2012 annual meeting.

Personal non-commercial use only. The Journal of Rheumatology Copyright @ 2014 . All rights reserved. 
J Rheumatol 2013;40:1413-8.

5. Tillett W, Jadon D, Shaddick G, Robinson G, Sengupta R, Korendowych E, et al. Feasibility, reliability and sensitivity to change of four radiographic scoring methods in patients with psoriatic arthritis. Arthritis Care Res 2014;66:311-7.

6. Coates LC, Fransen J, Helliwell PS. Defining minimal disease activity in psoriatic arthritis: a proposed objective target for treatment. Ann Rheum Dis 2010;69:48-53.
7. Winchester R, Minevich G, Steshenko V, Kirby B, Kane D, Greenberg DA, et al. HLA associations reveal genetic heterogeneity in psoriatic arthritis and in the psoriasis phenotype. Arthritis Rheum 2012;64:1134-44.

8. Eder L, Chandran V, Gladman DD. The Framingham Risk Score underestimates the extent of subclinical atherosclerosis in patients with psoriatic disease. Ann Rheum Dis 2013 July 25 (E-pub ahead of print). 